[4.0-16.0]; SLICC damage index score $1.0(0-2.0)$ and 28 healthy donors. Serum samples were analysed for SLE-associated ANA (anti-dsDNA, anti-Sm, anti-chromatin, anti-SS-A/Ro $52 \mathrm{kDa}$ and $60 \mathrm{kDa}$, anti-SS-B/La, anti-RNP-70, anti-ribosomal $\mathrm{P}$ - anti-RibP) using multiplex bead-based immunoassay system BioPlex 2200 (ANA Screen; Bio-Rad Laboratories Inc., USA). The levels of cytokines were determined with Bio-Plex 200 technology (Human Grp I Cytokine 27-plex panel; Bio-Rad Laboratories Inc., USA).

Results: Serum levels of anti-dsDNA, anti-chromatin, anti-Sm and anti-RibP antibodies were positively correlated with SLEDAI-2K scores $(r=0.6, r=0.7, r=0.4$, $r=0.3, p<0.05)$. Antibodies to dsDNA, chromatin, Sm and SS-A/Ro was most often detected in patients with dermatologic $(59 \%, 57 \%, 32 \%, 43 \%)$, renal $(64 \%, 66 \%$, $36 \%, 38 \%)$ and neuropsychiatric $(61 \%, 61 \%, 28 \%, 39 \%)$ manifestations of SLE. Increased serum concentrations of IFN-inducible chemokines: IP 10 and MCP1 were associated with high anti-dsDNA $(r=0.3)$, anti-chromatin $(r=0.5)$, anti-Sm $(r=0.5)$, anti-SS-B/La $(r=0.3)$, anti-RibP $(r=0.4)(p<0.05)$ and anti-Sm $(r=0.3)$, antiSS-B/La $(r=0.3)$, anti-RibP $(r=0.3)(p<0.05)$ antibodies levels. Hyperproduction of chemokines RANTES and IL-8/MIP- $1 \alpha$ negatively correlated with anti-dsDNA $(r=-0.3)$, anti-chromatin $(r=-0.3)(p<0.05)$ and anti-SS-A/Ro $(r=-0.3)(p<0.05)$ antibodies levels, respectively. Low levels of pro-inflammatory cytokines IL-1 $\beta$, IL15 were negatively associated with the presence of antibodies to dsDNA and $\operatorname{RibP}(r=-0.3)(p<0.05)$, but a positive correlation was found between the levels of anti-inflammatory cytokines IL-10, IL-1 ra and anti-Sm ( $r=0.3)$, anti-SS-B/La $(r=0.3)$, anti-SS-A/Ro $(r=0.3)(p<0.05)$ antibodies titers. Elevated concentration of GM-CSF was negatively correlated with serum level of antibodies to $\mathrm{Sm}(r=-0.3)$ $(p<0.05)$.

Conclusions: These data suggest that the presence of ANA clusters may identify patients with distinct clinical subtypes and cytokine profiles and can reflecting different pathways of immune dysregulation in SLE.

Disclosure of Interest: None declared

DOI: 10.1136/annrheumdis-2018-eular.6409

\section{AB1186 DYNAMIC CONTRAST ENHANCED MR IMAGING IN EARLY STAGE KNEE OSTEOARTHRITIS: A TEST- RETEST REPEATABILITY STUDY IN HEALTHY AND MODERATELY DISEASED SUBJECTS}

F. Sanaei Nezhad ${ }^{1}$, J. MacKay², J. Kaggie², M. Graves ${ }^{2}$, F. Gilbert ${ }^{2}$, A. McCaskie ${ }^{3}$, H. Flynn ${ }^{1}$, C. Roberts ${ }^{1}$, R. Janiczek ${ }^{4}$, A.R. Morgan ${ }^{4}$, G.J. Parker ${ }^{1,5} .{ }^{1}$ Bioxydyn, Manchester, ${ }^{2}$ Department of Radiology; ${ }^{3}$ Trauma and Orthopaedics, University of Cambridge, Cambridge; ${ }^{4} \mathrm{GSK}$, Stevenage; ${ }^{5}$ Centre for Imaging Sciences, University of Manchester, Manchester, UK

Background: Osteoarthritis $(\mathrm{OA})$ in the knee exhibits signs of synovial activation in early phase of disease $\left(.{ }^{1}\right.$ DCE-MRI provides quantitative measurement of vascular disruption associated with synovitis $\left({ }^{2,3}\right.$ and has been shown to be sensitive to early treatment-induced changes in small group sizes in multicentre trials $\left(.{ }^{4}\right.$ Objectives: 1. Determine the reproducibility of DCE-MRI parameters in the knee. 2. Compare DCE-MRI parameters between participants with mild/moderate OA and aged matched controls.

3. Enable effect and sample size calculations for further studies.

Methods: 9 knee OA patients and 4 controls underwent two MRI scans with a month separation. All patients had diagnosis of knee OA as per ACR criteria, with medial compartment predominant disease and Kellgren-Lawrence grade 2/3 on radiographs. 3 pre-contrast injection series were obtained with flip angles $2^{\circ}, 6^{\circ}$ and $14^{\circ}$ for $\mathrm{T} 1$ calculation. 35 consecutive phases formed the DCE-MRI series (temporal resolution:14 s). Contrast agent(CA)(Dotarem $(0.4 \mathrm{~mL} / \mathrm{kg})$ )was administrated on the 6 th phase at the rate $3 \mathrm{~mL} / \mathrm{s}$ followed by $50 \mathrm{~mL}$ saline. All Images were registered together. Here 2 DCE paramaters are reported: $K^{\text {rans }}\left(\min ^{-1}\right)$, volume transfer constant for CA between blood plasma and extravascular extracellular space; estimated using the extended Tofts model $\left({ }^{5}\right.$ with population arterial function $\left(;{ }^{6}\right.$ and IAUC 60 (mM.s), initial area under CA concentration curve over $60 \mathrm{~s}$ post-arrival. Manual segmentation was performed by radiologist.

Results: Test-retest coefficient of variation(CoV) was lower in controls. $K^{\text {trans }}$ had CoVs of $26 / 16 \%$ in OA patients/controls. An effect size of $30 \%$ reduction in $K^{\text {rans }}\left(,{ }^{4}\right.$ indicates a treatment effect would be observed in a group of 11 patients with $90 \%$ power. DCE parameters were generally higher in OA patients than in controls. 4 out of 9 patients (All with KL score 3)demonstrated parameter values outside the healthy volunteer range at $\mathrm{p}<0.01$ level (one sample t-test).

\begin{tabular}{lccccc}
\multicolumn{1}{l}{ Abstract AB1186 - Table 1 } & $\begin{array}{c}\text { Age } \\
(\text { mean } \\
\pm S D)\end{array}$ & $\begin{array}{c}\text { BMl } \\
(\text { mean } \\
\pm S D)\end{array}$ & $\begin{array}{c}\text { \#KL- } \\
\text { grade 2/ } \\
3\end{array}$ & $\begin{array}{c}\text { \#MOAKS effusion } \\
\text { synovitis grade 0/1/2 }\end{array}$ & $\begin{array}{c}\text { \#MOAKS Hoffa } \\
\text { synovitis grad 0/1 }\end{array}$ \\
\hline $\begin{array}{l}\text { Patient } \\
(\mathrm{n}=9,4 \mathrm{~F})\end{array}$ & $52 \pm 5.2$ & $30 \pm 4.15$ & $5 / 4$ & $5 / 1 / 3$ & $7 / 2$ \\
$\begin{array}{l}\text { control } \\
(\mathrm{n}=4,3 \mathrm{~F})\end{array}$ & $55 \pm 3.0$ & $30 \pm 3.50$ & NA & $3 / 1 / 0$ & $3 / 1$ \\
\hline
\end{tabular}
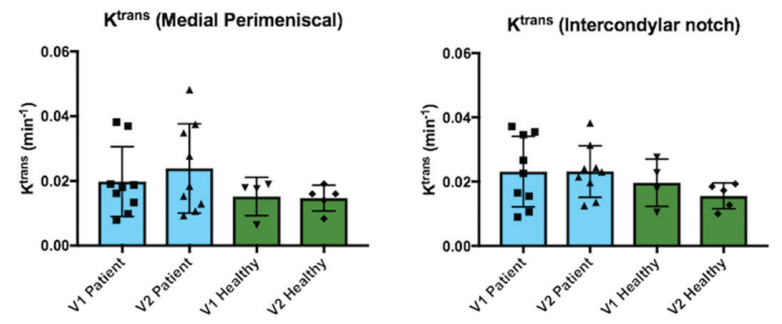

IAUC $_{60}$ (Medial Perimeniscal)

IAUC $_{60}$ (Intercondylar notch)
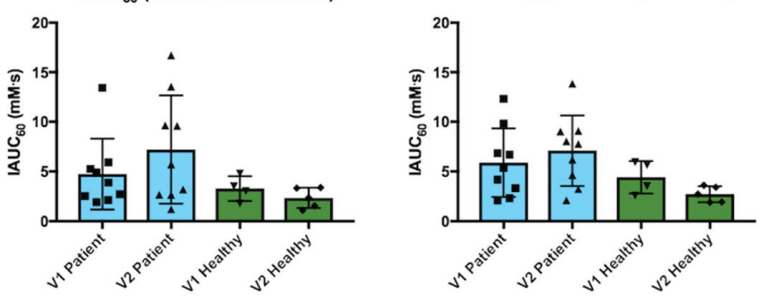

Abstract AB1186 - Figure 1

Conclusions: Higher $K^{\text {trans and }} \mathrm{IAUC}_{60}$ in patients indicates the potential of revealing microvascular function differences. The fact that this is not observed in all patients could suggest phenotypical variation. High CoV values in patient relative to healthy may be explained in part by fluctuation in disease status. Manual delineation also contributes to this variation.

DCE-MRI measures of vascular disruption associated with synovitis in knee OA are practical and feasible for imaging trials. These measures offer greater insight and sensitivity into the inflammatory component of OA that is not captured using other radiological methods.

\section{REFERENCES:}

[1] Roemer, et al. Osteoarthr Cartil.2010;1269-74.

[2] Wenham, et al. Osteoarthr Cartil.2014; 1614-18.

[3] Gait, et al. Osteoarthr. Cartil 2016;1392-8.

[4] Waterton, et al. Eu. Rad 2017;3662-8.

[5] Tofts, et al. JMRI.1999;223-32.

[6] Parker, et al MRM 2006:993-1000.

Acknowledgements: National Institute for Health Research Cambridge Biomedical Research Centre.

Funding provided by GlaxoSmithKline.

Disclosure of Interest: None declared

DOI: 10.1136/annrheumdis-2018-eular.6429

\section{AB1187 WHAT IMAGING DETECTED PATHOLOGIES ARE ASSOCIATED WITH SHOULDER SYMPTOMS AND THEIR PERSISTENCE? A SYSTEMATIC LITERATURE REVIEW}

G. Tran ${ }^{1}$, P. Cowling ${ }^{2}$, T. Smith ${ }^{3}$, A. Lucas ${ }^{1}$, E. Hensor ${ }^{1}$, A. Barr ${ }^{1}$, S. Kingsbury ${ }^{1}$, P. Conaghan ${ }^{1} .^{1}$ Leeds Institue of Rheumatic and Musculoskeletal Medicine; ${ }^{2}$ LEEDS TEACHING HOSPITAL TRUST, Leeds; ${ }^{3}$ Nuffield Department of Orthopaedics, Rheumatology and Musculoskeletal Sciences, Oxford, UK

Background: Shoulder pain is a very common musculoskeletal complaint and a significant contributor to disability and morbidity. Recovery can be slow and ove $50 \%$ continue to have pain at 18 months. Shoulder pain has a significant negative impact on quality of life and poses a significant economic burden, with costs estimated to be $€ 345$ million per year in the UK alone. Modern imaging modalities can accurately detect soft-tissue pathologies and are increasingly used, but the relationship of imaging findings to patient symptoms remain unclear.

Objectives: Our aim was to systematically review the literature to determine what imaging features are associated with symptoms and their progression.

Methods: A systematic review using Medline, EMBASE, Cochrane and grey literature was conducted to April 2017. The cross-sectional and longitudinal relationships between imaging-detected abnormalities and symptoms were analysed and associations qualitatively characterised by a best evidence synthesis based on study design, covariate adjustment and the Grade of Recommendations 